\title{
Initial value problems spreadsheet solver using VBA for engineering education
}

\author{
Çiğdem Dinçkal ${ }^{\mathrm{a}^{*}}$ \\ ${ }^{\text {a }}$ Cankaya University, Ankara, Turkey \\ *Corresponding authorE-mail: cdinckal@cankaya.edu.tr
}

\begin{abstract}
Article Info
Keywords: Excel spreadsheet, Initial Value Problems (IVPs) spreadsheet solver, Runge-Kutta methods, VBA programming

2010 AMS: $65 L X X, \quad 65 Y X X$, 68WXX,68UXX, $68 N X X$

Received: 4 March 2018

Accepted: 22 April 2018

Available online: 30 June 2018
\end{abstract}

Abstract

Spreadsheet solver using VBA programming has been designed for solving initial value problems (IVPs), analytically and numerically by all Runge-Kutta (RK) methods including also fifth order with calculation of true percent relative error for corresponding RK method. This solver is user-friendly especially for beginner users of Excel and VBA.

\section{Introduction}

IVPs arise in any field of science and engineering education such as mechanics, geotechnics, dynamics, chemical kinetics, optimization and stability, et cetera. There are computing approaches; exact solution method and numerical methods for solving these IVPs. Numerical methods are both applicable and practical in solving IVPs in many engineering problems because of the existence of complicated problems in engineering and limitations of exact solution method $[1,2]$. Numerical methods yield approximate the solutions of the IVPs, particularly for the nonlinear IVPs.

This study mainly has focussed on numerical solutions followed by Euler and various Runge-Kutta methods for solving single IVPs. These methods progress the solution over step starting from some given initial condition at the initial starting point. To simplify the steps in solving IVPs by RK methods, a tool is used. This tool is a prevalent spreadsheet application, fundamentally called as Excel, also commonly used by professionals for diverse applications in business [3], engineering and science [4]-[6].

Numerical methods in science and engineering may also be implemented in by use of Excel and also VBA. Use of VBA in explicit form Visual Basic for Applications programming capability lurks in the background behind Excel handled in the texts like Lilley and Chapra [2, 7]. In addition to this, a series of studies in literature employed spreadsheet as a calculator or solver to focus on design of solver and calculator for polynomial interpolation [8,9], solution for systems of linear and nonlinear equations [10, 11], computation of eigenvalues [12, 13], design of spreadsheet calculator for numerical differentiation [14]-[16], spreadsheet solver for solution of partial differential equations [17], a spreadsheet solution of system of initial value problems using fourth-order RK method [18], and fourth-order RK method by spreadsheet [19]. Only the works of Tay et al. [20, 21] include design of spreadsheet calculator for solving system of IVPs using fourth-order RK method and also solving IVPs using fourth-order RK method with use of VBA programming.

In this study, a spreadsheet solver is designed to solve both IVPs by all RK methods and also exact solution method in the spreadsheet environment based on VBA programming. Microsoft Excel 2010 and Microsoft Visual Basics for Applications 7.0 are used during this study. The generation of VBA programming includes three steps. The first step is to develop an user interface input form is designed to acquire the needed information such as initial conditions of independent and dependent variables for each RK method, step size and number of steps. Then a general VBA code for any IVPs is created behind the Solve button in user interface input form. The third step is to generate function files depending on the related IVP and its analytical solution. Once the SOLVE button in user interface input form is clicked, the complete numerical and analytical solutions of the IVP and corresponding true percent relative error will be computed automatically for each order of RK method. 
Examples are presented from various fields of engineering to demonstrate the merits of this unconventional solver design which shields the tedious algorithmic implementation details from the user (such as students and educators) and greatly simplifies solving an IVP using RKSOLVER.

This spreadsheet solver is user-friendly such that users only require to enter initial conditions of independent and dependent variables for each RK method, step size and number of steps at the first step to compute the complete solution of the IVPs automatically without typing any commands in the spreadsheet cells. Here, complete solution of the IVPs means solutions from each order of RK method, exact solutions and also true percent relative errors in terms of comparison with each RK method and exact solutions. So users as educators have an oppurtunity to elucidate students the differences and similarities that exist between each order of RK method and also exact solutions at the same time and be able to comment on the solution of any engineering problem including IVPs correctly. There is no need to know the various derivations of RK methods and memorize the complicated formulations of RK methods. The solver is general and standard for any engineering problem. The main aim of this paper is to design a tool in other words spreadsheet solver which employes both numerical methods: RK methods with fifth order and also analytical methods giving exact solutions with automatically calculated true percent relative errors in solving IVPs at the same time. Therefore this solver is called as IVP spreadsheet solver.

\section{Runge Kutta (RK) methods}

This section is devoted to solving IVPs of the form given below:

$$
\frac{d y}{d x}=f(x, y)
$$

with the initial value $y\left(x_{0}\right)=y_{0}$ for the number of points $n$ within the interval $x_{0} \leq x \leq x_{n}$. Here $x$ is the independent variable, $y$ is the dependent variable, $f$ is the function of derivation (in other words slope) and $h$ is the fixed step size. $n$, the number of steps can be found as $\left(x_{n}-x_{0}\right) / h[1]$.

\section{1) First-Order RK Method}

\section{Euler's Method:}

$$
y_{i+1}=y_{i}+h k_{1}
$$

where $k_{1}=f(x, y)$

\section{2) Second-Order RK Methods}

a) Heun's Method:

$$
y_{i+1}=y_{i}+h\left(\frac{k_{1}+k_{2}}{2}\right)
$$

where $k_{2}=f\left(x_{i}+h, y_{i}+h k_{1}\right)$

b) Midpoint (Improved Polygon) Method:

$$
y_{i+1}=y_{i}+h k_{2}
$$

where $k_{2}=f\left(x_{i}+\frac{h}{2}, y_{i}+\frac{k_{1} h}{2}\right)$

c) Ralston's Method:

$$
y_{i+1}=y_{i}+\left(\frac{k_{1}+2 k_{2}}{3}\right) h
$$

where $k_{2}=f\left(x_{i}+\frac{3 h}{4}, y_{i}+\frac{3 h k_{1}}{4}\right)$

\section{3) Third-Order RK Method}

$$
y_{i+1}=y_{i}+\left(\frac{k_{1}+4 k_{2}+k_{3}}{6}\right) h
$$

where $k_{2}=f\left(x_{i}+\frac{h}{2}, y_{i}+\frac{k_{1} h}{2}\right), \quad k_{3}=f\left(x_{i}+h, y_{i}-k_{1} h+2 k_{2} h\right)$

4) Fourth-Order RK Method

$$
y_{i+1}=y_{i}+\left(\frac{k_{1}+2 k_{2}+2 k_{3}+k_{4}}{6}\right) h
$$




\begin{tabular}{|l|}
\hline $\begin{array}{l}\text { Function } \mathrm{f}(\mathrm{x}, \mathrm{y} 0, \mathrm{~h}) \\
\mathrm{f}=\mathrm{y} 0 / 0.2254\end{array}$ \\
\hline End Function \\
\hline
\end{tabular}

Table 1: Function module for stress-strain relationship IVP

Function $\operatorname{fexact}(\mathrm{x}, \mathrm{y} 0, \mathrm{~h}, \mathrm{i})$
fexact $=\operatorname{Exp}((\mathrm{h} * \mathrm{i}) / 0.2254)$
End Function

Table 2: Function module for exact solution of stress-strain relationship

where $k_{2}=f\left(x_{i}+\frac{h}{2}, y_{i}+\frac{k_{1} h}{2}\right), \quad k_{3}=f\left(x_{i}+\frac{h}{2}, y_{i}+\frac{k_{2} h}{2}\right), \quad k_{4}=f\left(x_{i}+h, y_{i}+k_{3} h\right)$

\section{5) Fifth-Order RK Method}

$$
y_{i+1}=y_{i}+\left(\frac{7 k_{1}+32 k_{3}+12 k_{4}+32 k_{5}+7 k_{6}}{90}\right) h
$$

where $k_{2}=f\left(x_{i}+\frac{h}{4}, y_{i}+\frac{k_{1} h}{4}\right), \quad k_{3}=f\left(x_{i}+\frac{h}{4}, y_{i}+\frac{k_{1} h}{8}+\frac{k_{2} h}{8}\right), \quad k_{4}=f\left(x_{i}+\frac{h}{2}, y_{i}-\frac{k_{2} h}{2}+k_{3} h\right), \quad k_{5}=f\left(x_{i}+\frac{3 h}{4}, y_{i}+\frac{3 k_{1} h}{16}+\frac{9 k_{4} h}{16}\right)$, and $k_{6}=f\left(x_{i}+h, y_{i}-\frac{3 k_{1} h}{7}+\frac{2 k_{2} h}{7}+\frac{12 k_{3} h}{7}-\frac{12 k_{4} h}{7}+\frac{8 k_{5} h}{7}\right)$

It should be noted that k's are recurrence relationships. In other words, $k_{1}$ appears in the equation for $k_{2}$ which appears in the equation for $k_{3}$ and so on. Since each $k$ is a functional evaluation, this recurrence makes RK methods efficient for computations [1].

In this work, fifth-order RK method yields the superior results in terms of less error than the other order of RK methods. As the order of RK method increases, convergence to the exact results also increases in terms of less errors.

\section{Numerical examples}

Numerical examples are presented from various engineering applications.

1) Geotechnical Engineering

To mIVPl the the behavior of soil under the effect of load, it is required to formulate the stress and strain relationship and this is achieved by the following IVP:

$$
\frac{d \sigma}{d \varepsilon}=\frac{\sigma}{c_{C}}
$$

The exact solution for equation (3.1) is

$$
\sigma=e^{\frac{\varepsilon}{c_{C}}}
$$

where $\sigma$ is the stress, $\varepsilon$ is the strain of soil and $c_{C}$ is the compression index and it is 0.2254 for this soil type. Initial conditions are, $\varepsilon_{0}$ is 0 for independent variable and $\sigma_{0}$ is $1 \mathrm{kPa}$ for dependent variable. Final $\varepsilon$ is 1.2 and step size (h) is 0.1 . This means that number of steps (n) is 12 . At first, for each numerical example, function modules are prepared for both IVP and exact solution of it respectively. These modules change from example to example. The functions for IVP and exact solution are illustrated in the following tables.

Here $\mathrm{x}$ is the independent variable, $\mathrm{y} 0$ is the initial dependent variable, $\mathrm{i}$ is the counter of steps.

Then equations (2.2) to (2.8) are applied to obtain the solutions by each order of RK method respectively. Besides exact solution of the IVP with true percent relative error for each RK method are also incorporated in the computations.

Finally IVP spreadsheet solver is applied which is discussed in the next section to obtain the complete solutions.

\section{2) Mechanical Engineering}

To determine the change in velocity in other words acceleration of a free-falling body to the forces acting on it with considering the air resistance, the following IVP is used:

$$
\frac{d v}{d t}=g-\frac{c}{m} v
$$

The exact solution for equation (3.3), which also gives velocity of the object, is

$$
v(t)=\frac{g m}{c}\left(1-e^{\left(-\frac{c}{m}\right) t}\right)
$$

where $v$ is the velocity (dependent variable $y$ ), $t$ is the time in seconds (indepedent variable $x$ ), $g$ is the gravitational constant, $9.8 \mathrm{~m} / \mathrm{s}^{2}, \mathrm{~m}$ is the mass of the object, $68.1 \mathrm{~kg}$ and $\mathrm{c}$ is the drag coefficient, $12.5 \mathrm{~kg} / \mathrm{s}$. Initial conditions are, $t_{0}$ is $0 \mathrm{~s}$ and $v_{0}$ is $0 \mathrm{~m} / \mathrm{s}$ [1]. Final value of time is $5 \mathrm{~s}$ and step size (h) is 0.5 . This means that number of steps (n) for computation is 10 .

At first, for this example, function modules are written for both IVP and exact solution of it respectively. These functions are illustrated in Table 3 and Table 4 respectively.

Here $\mathrm{x}$ is the independent variable corresponding to time, $\mathrm{y} 0$ is the initial dependent variable corresponding to velocity. 


Function $\mathrm{f}(\mathrm{x}, \mathrm{y} 0, \mathrm{~h})$
$\mathrm{f}=9.8-((12.5 / 68.1) * \mathrm{y} 0)$
End Function

Table 3: Function module for exact solution yielding velocity

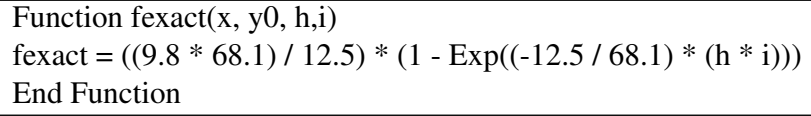

Table 4: Function module for exact solution yielding velocity

Like geotechnical engineering example, equations (2.2) to (2.8) are employed to find the solutions by each order of RK method respectively. Besides exact solution of the IVP with true percent relative error for each RK method are also inserted in the computations.

Finally IVP spreadsheet solver is used which is mentioned in the next section to obtain the complete solutions.

\section{3) Chemical Engineering: Mixture Problem}

The mixture problem related to a tank containing $1000 \mathrm{~L}$ of brine with $15 \mathrm{~kg}$ of dissolved salt. Pure water enters the tank at a rate of 10 $\mathrm{L} / \mathrm{min}$. The solution is kept thoroughly mixed and drains from the tank at the same time. In this problem, it is required to determine the amount of salt after $t$ minutes in this tank. For this reason, the following IVP is employed:

$$
\frac{d A}{d t}=\frac{-A}{100}
$$

$A(t) \quad$ is the amount of salt after $t$ minutes in tank, also the dependent variable is obtained by the following exact solution:

$$
A(t)=15 e^{\left(\frac{-t}{100}\right)}
$$

Initial conditions are, $t_{0}$ is $0 \mathrm{~min}$ and $A_{0}$ is $15 \mathrm{~kg}$. Final value of time is $0.96 \mathrm{~min}$ and step size (h) is 0.02 . Number of steps (n) for computation is 49 .

At first, function modules are formed for both IVP and exact solution of the problem respectively. These functions are displayed in Table 5 and Table 6 respectively.

Here $\mathrm{x}$ is the independent variable corresponding to time, $\mathrm{y} 0$ is the initial dependent variable corresponding to amount of salt after $\mathrm{t}$ minutes in the tank.

Then, equations (2.2) to (2.8) are used to determine the solutions by writing codes for each order of RK method respectively. These codes are standard and valid for any scince and engineering problem including IVP. So there is no need to write cIVP for various problems. Besides exact solution of the IVP with true percent relative error for each RK method are also included in the computations. True percent relative error is in the following form:

$$
\varepsilon_{T}=\left|\frac{\text { ExactResult }- \text { ApproximateResult }}{\text { ExactResult }}\right| \times 100
$$

Where Exact Result in other words true result represents the solution obtained by analytically. Approximate Result corresponds with the corresponding solution obtained by numerical methods, any order of RK methods.

Finally IVP spreadsheet solver is employed which is argued in the next section to obtain the complete solutions.

\section{IVP spreadsheet solver}

Using this IVP spreadsheet solver leads to a macro named RKSOLVER which solves the whole IVP at once completely.

The general procedure for obtaining complete solution of an IVP is composed of some steps. These steps are standard and applicable for any type of IVP.

The first step is to design an user interface input form (userform) called as UserForm4 to enable users to enter required data for solving an IVP completely. The standard form of UserForm4 for any problem is illustrated in Figure 4.1.

The second step is to generate a new tab name as IVP Solver with RKSOLVER macro including codes for solving IVP by both numerically (by each order of RK method) and analytically (gives exact solution). RKSOLVER also provides user to compute true percent relative error for each RK method.

Figure 4.2 illustrates the standard IVP Solver tab with RKSOLVER button. One more variation is to add a button assigned RKSOLVER macro in the spreadsheet. So user is able to run the macro simply by clicking this button. It is sufficient to start the complete solution procedure of IVPs.

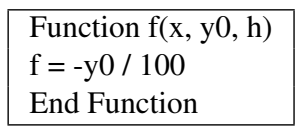




\begin{tabular}{l}
\hline Function fexact(x, y0, h, i) \\
fexact $=15 * \operatorname{Exp}(-(\mathrm{h} * \mathrm{i}) / 100)$ \\
End Function
\end{tabular}

Table 6: Function module for exact solution of the problem

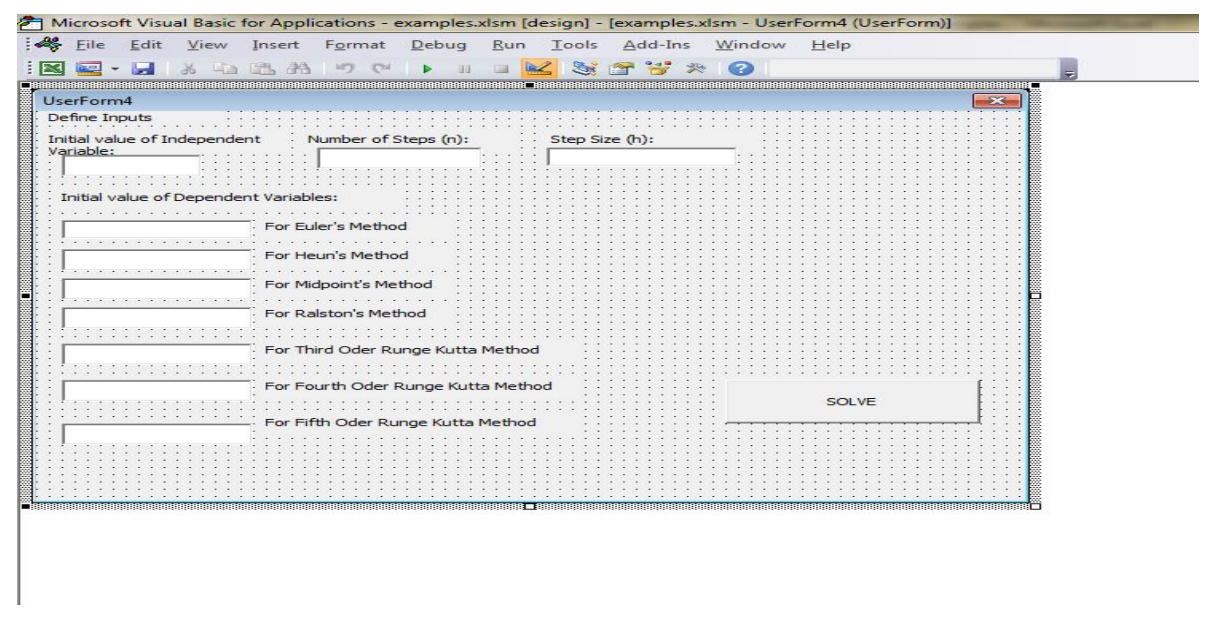

Figure 4.1: The standard userform for all examples

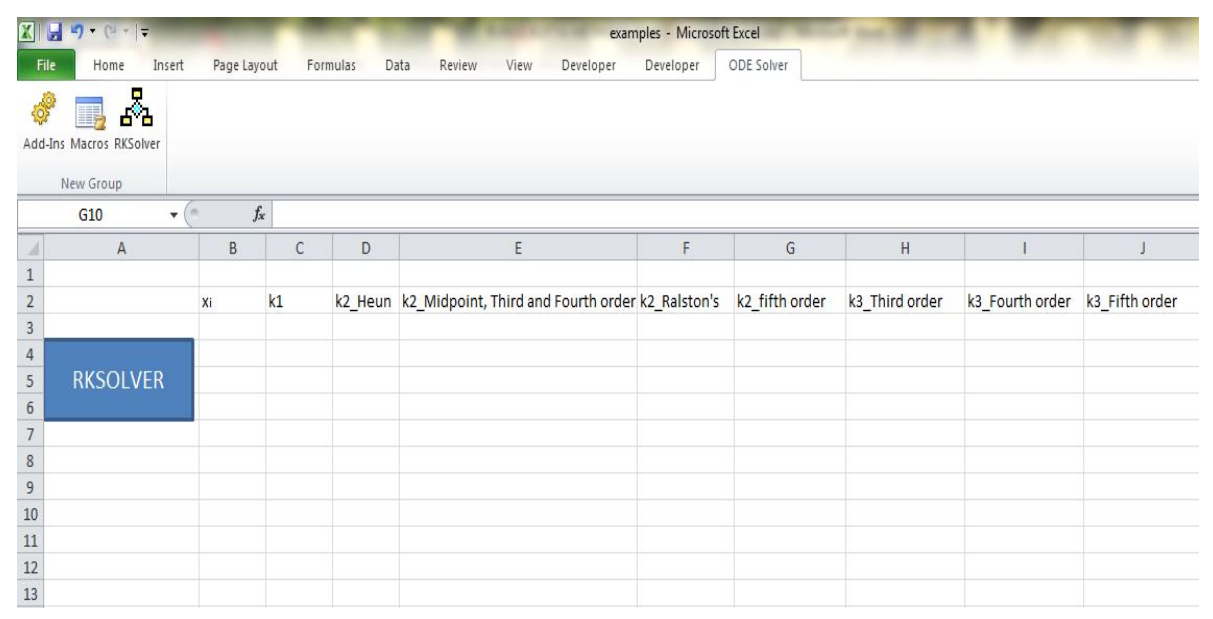

Figure 4.2: The standard IVP Solver tab with RKSOLVER button

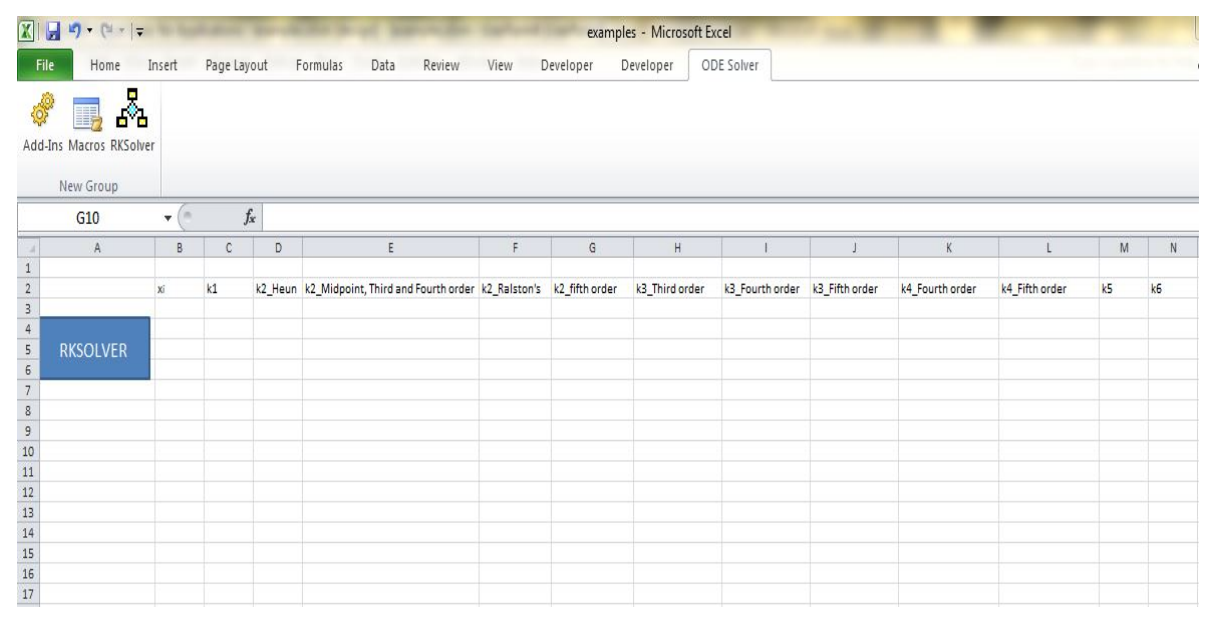

Figure 4.3: The standard blank spreadsheet image with k's (recurrence relationships) titles 


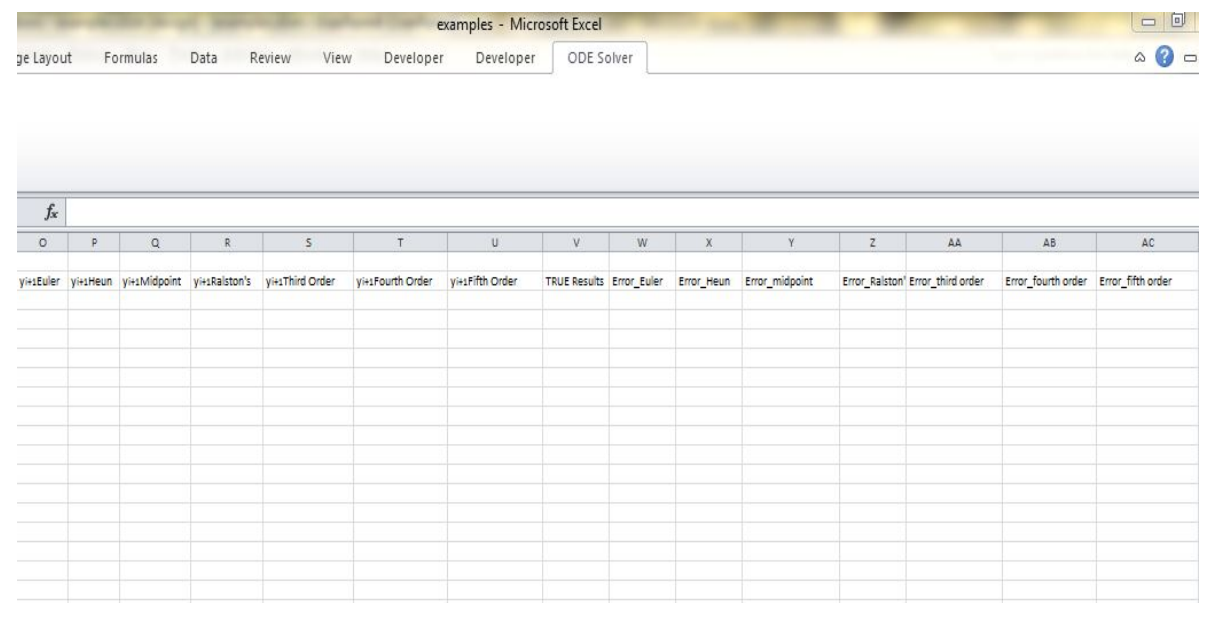

Figure 4.4: The standard blank spreadsheet image with RK results, exact results and error titles

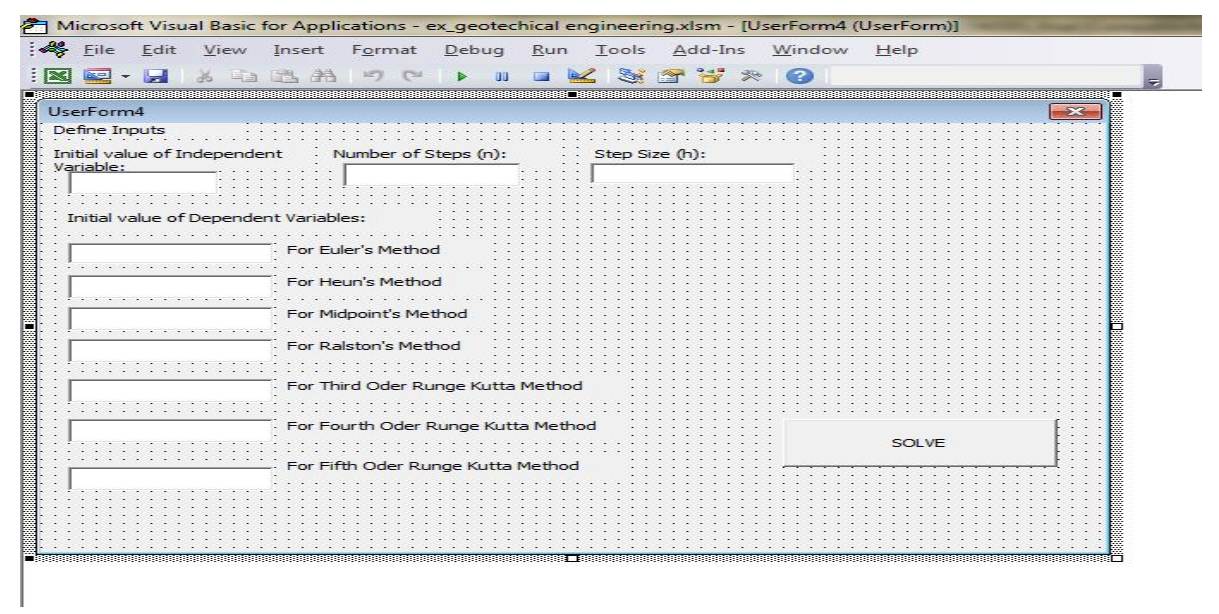

Figure 4.5: Userform for geotechnical engineering example

Then the only thing is to specify sufficient place in spreadsheet cells to make macro fill them with solutions for any IVP examples. For this reason, the titles for k's, RK results, exact results and error titles are written as is the case with Figure 4.3 and Figure 4.4 respectively.

The working procedure for IVP solver namely RKSOLVER is described for each numerical examples (geotechnical engineering, mechanical engineering and chemical engineering). The steps for geotechnical engineering example are illustrated in the Figure 4.5- Figure 4.11.

The first step is to call userform by clicking run in the toolbar or simply clicking RKSOLVER button. The image of this userform for geotechnical engineering example is given in Figure 4.5. This userform is standard for any IVP example.

Due to the fact that initial conditions are different for all IVPs, the filled userform is distinctive for all problems. As is the case with geotechnical engineering example. Userform is filled with initial conditions of the problem in Figure 4.6. Then by clicking SOLVE button in UserForm4; k's, numerical solutions obtained form all RK methods, exact solutions (true solutions) and true percent relative errors can be obtained and displayed as the spreadsheet images in Figure 4.7 to Figure 4.11 respectively.

To Figure 4.10 and Figure 4.11, fifth-order RK method gives the best solution in terms of the least error and best convergence to exact solutions.

Similarly for mechanical engineering, userform is invoked by clicking RKSOLVER in Figure 4.12. Then this form is filled with necessary data as it is shown in Figure 4.13.

By clicking the SOLVE button in userform, computations are performed and given in the spreadsheet images of Figure 4.14 to Figure 4.18. To Figure 4.17 and Figure 4.18, the worst solution is obtained by Euler's method while fifth-order RK method is the best one with the least error and best convergence to the exact solution.

For mixture problem, userform is called by clicking RKSOLVER button in spreadsheet. Figure 4.19 illustrates this process.

Then this userform is filled by entering initial conditions as given in Figure 4.20. Clicking the SOLVE button in userform leads to complete solution of the problem. These solutions are displayed in Figure 4.21 to Figure 4.25.

To Figure 4.24 and Figure 4.25, all RK methods give quite well solutions with convergence to exact results in terms of less errors.

\section{Conclusion}

An IVP solver with use of RK methods including also the highest order; fifth order has been generated by VBA for the first time in literature. Emphasis was on all types of RK methods usable simultaneously and the solver generated applicable to IVPs for science and engineering problems. 


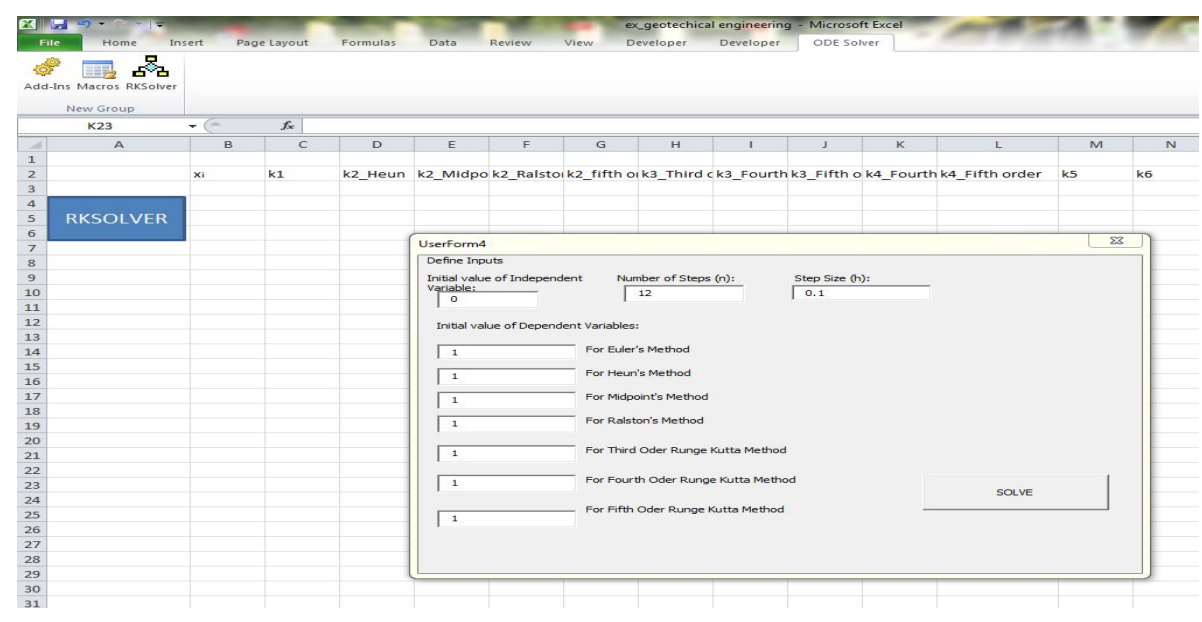

Figure 4.6: Filled userform for geotechnical engineering example

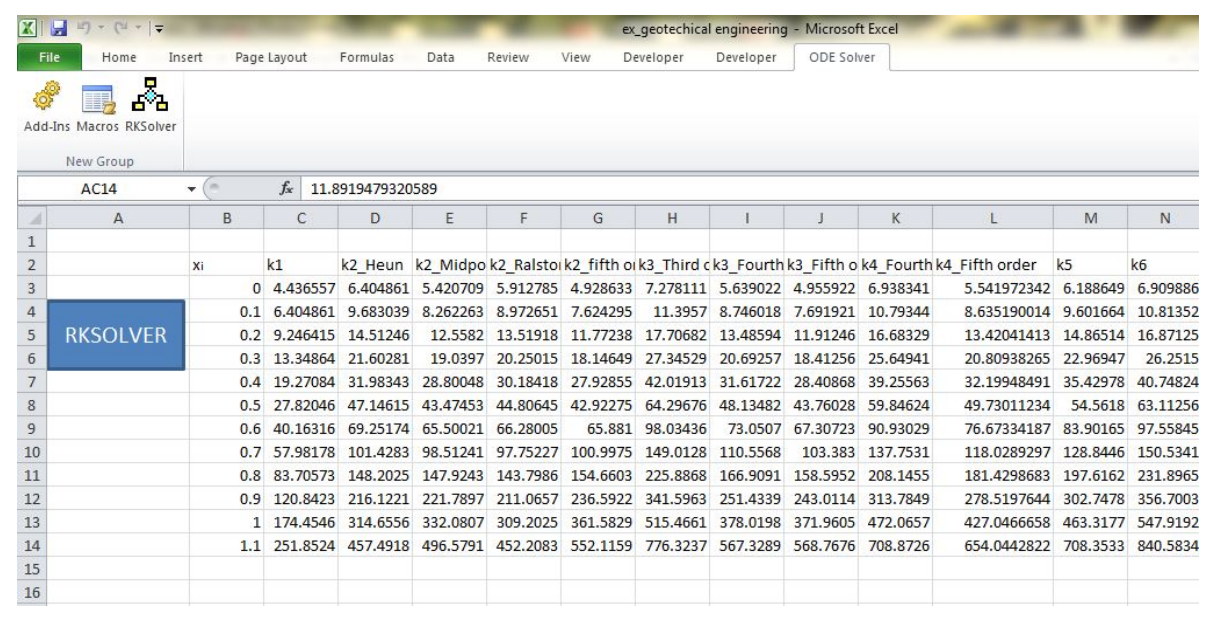

Figure 4.7: Computation results for k's for geotechnical engineering example

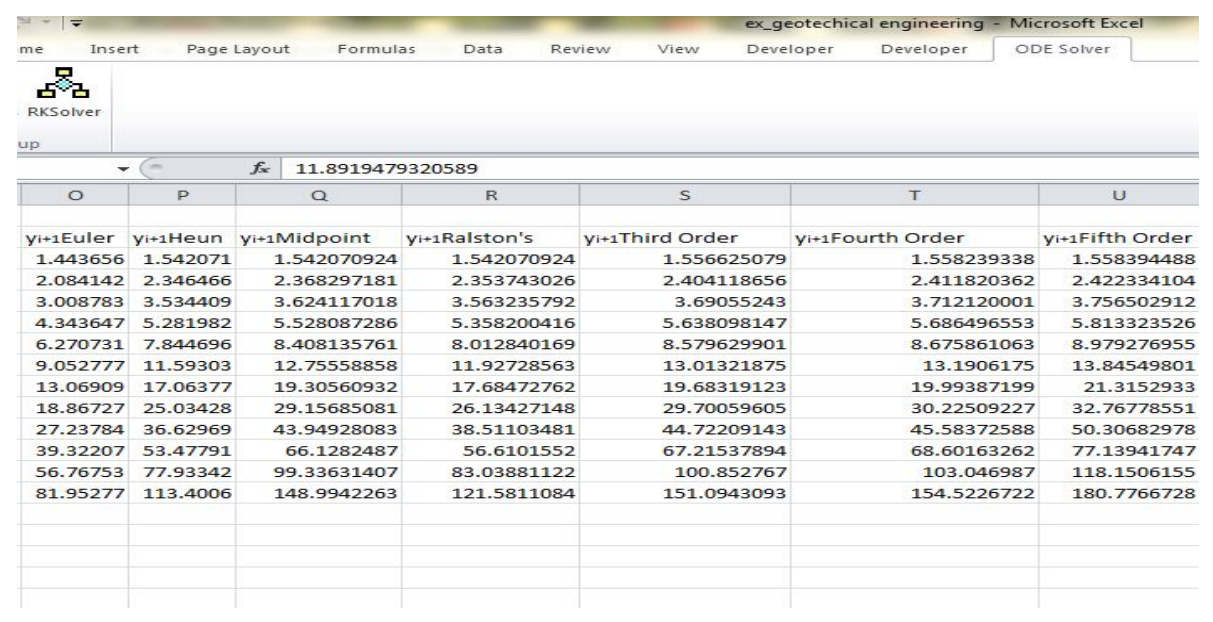

Figure 4.8: Computation results for each RK method for geotechnical engineering example 


\begin{tabular}{|c|c|c|c|c|c|c|c|}
\hline Formulas Data & Review View & $\begin{array}{l}\text { ex_geotechical } \\
\text { Developer }\end{array}$ & $\begin{array}{l}\text { I engineering - Microsoft Ex } \\
\text { Developer ODE Solver }\end{array}$ & Excel & -3 & $=0$ & $\begin{array}{l}\text { 口回 } 23 \\
00 \text { 口通 }\end{array}$ \\
\hline \multicolumn{8}{|l|}{ 3919479320589 } \\
\hline v & w & $x$ & Y & $z$ & AA & $A B$ & $A C$ \\
\hline IRUE Results & Error_Euler & Error_Heun & Error_midpoint & Error_Ralston's & Error_third order & Error_fourth order & Error_fifth order \\
\hline 1.558393874 & 7.362589976 & $\begin{array}{ll}6 & 1.047421393\end{array}$ & 1.047421393 & 1.047421393 & 0.113501165 & 0.009916402 & 3.93667E-05 \\
\hline 2.428591468 & 14,18310264 & $\begin{array}{ll}4 & 3.381611137\end{array}$ & 2.482685413 & 3.081969229 & 1.007695683 & 0.690569245 & 0.257654038 \\
\hline 3.784702066 & 20.50144892 & $2 \quad 6.613271635$ & 4.243003701 & 5.851617122 & 2.487636662 & 1.917774866 & 0.745082536 \\
\hline 5.898056516 & 26.35460128 & $8 \quad 10.44538333$ & 6.27273119 & 9.153118461 & 4.407525912 & 3.586943647 & 1.436625617 \\
\hline 9.191495146 & 31.77681002 & $2 \quad 14.6526716$ & 8.522654606 & 12.82332154 & 6.656863059 & 5.609904312 & 2.308853858 \\
\hline 14.32396973 & 36.79980377 & $7 \quad 19.06555263$ & 10.94934701 & 16.73198244 & 9.150752258 & 7.912277461 & 3.340356939 \\
\hline 22.32238668 & 41.45297508 & $\begin{array}{ll}8 & 23.55758767\end{array}$ & 13.51458252 & 20.77582085 & 11.82308816 & 10.43129807 & 4.511584689 \\
\hline 34.78707067 & 45.76355247 & $7 \quad 28.03568497$ & 16.18480588 & 24.87360685 & 14.62173883 & 13.11400561 & 5.804700193 \\
\hline 54.21195784 & 49.75675972 & $2 \quad 32.43245311$ & 18.93065186 & 28.96210293 & 17.50511655 & 15.91573575 & 7.20344407 \\
\hline 84.48358301 & 53.45596349 & $\begin{array}{l}9 \\
9\end{array}$ & 21.72651024 & 32.99271506 & 20.43971557 & 18.79885987 & 8.693009074 \\
\hline 131.6586982 & 56.88281006 & $6 \quad 40.80648133$ & 24.55013198 & 36.92873139 & 23.3983259 & 21.73172879 & 10.25992425 \\
\hline 205.1761088 & 60.05735196 & $6 \quad 44.7301015$ & 27.38227316 & 40.74304797 & 26.35872171 & 24.68778502 & 11.89194793 \\
\hline & & & & & & & \\
\hline & & & & & & & \\
\hline & & & & & & & \\
\hline & & & & & & & \\
\hline
\end{tabular}

Figure 4.9: Computation results for exact results (true results) and true percent relative errors of each RK method for geotechnical engineering example

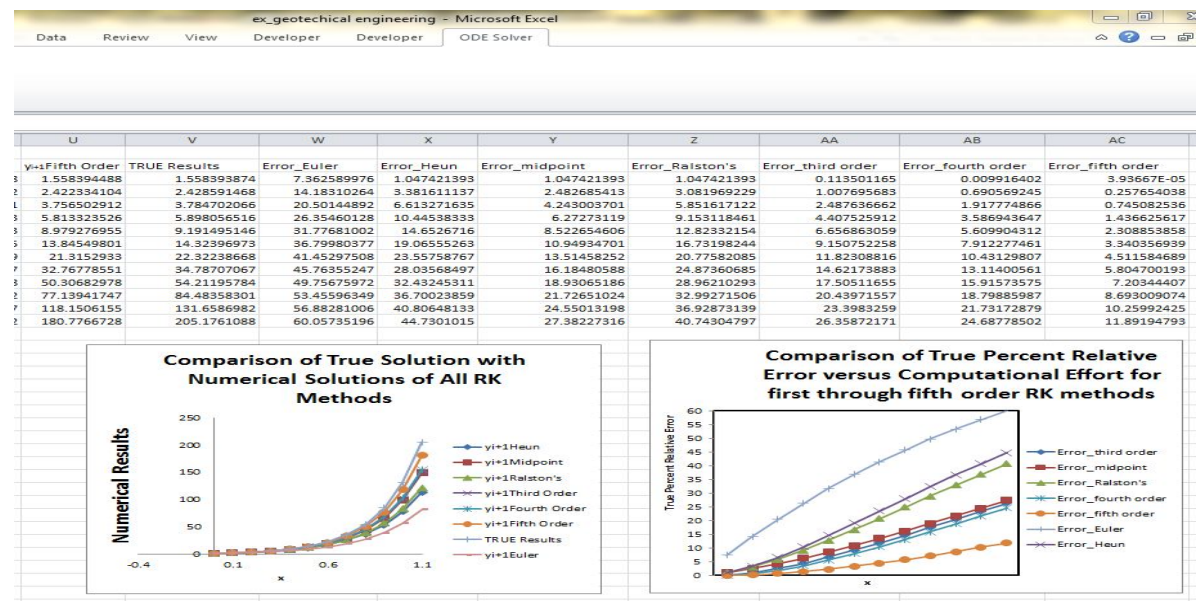

Figure 4.10: Graphical display of the computation results for geotechnical engineering example

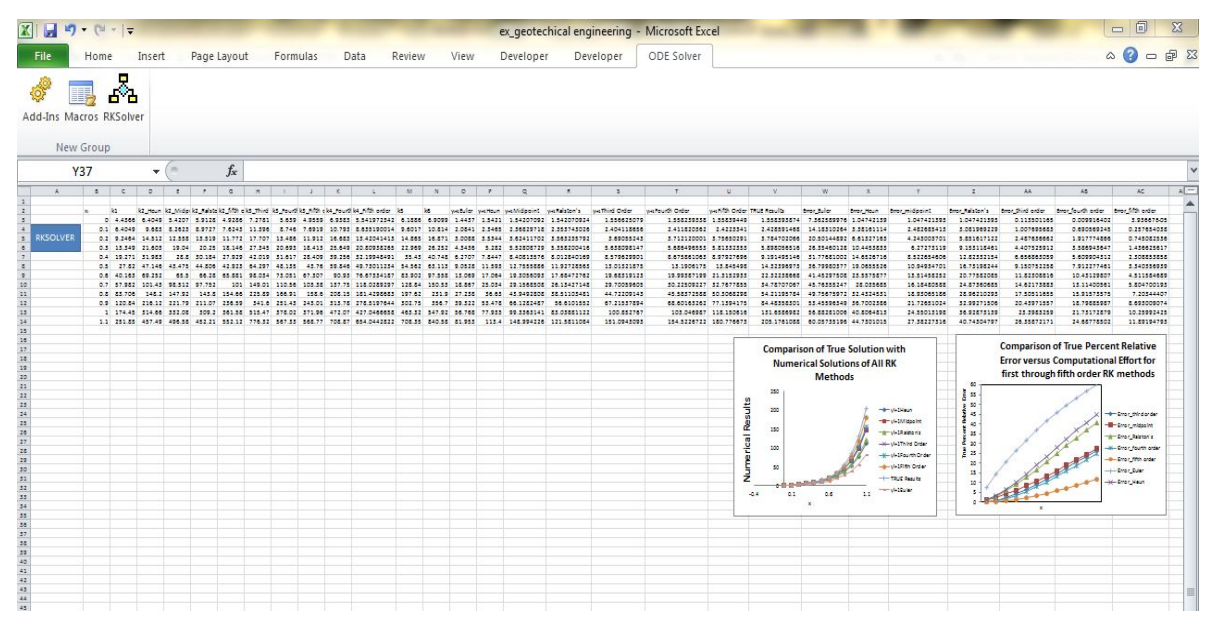

Figure 4.11: The spreadsheet image of full computation results for geotechnical engineering 


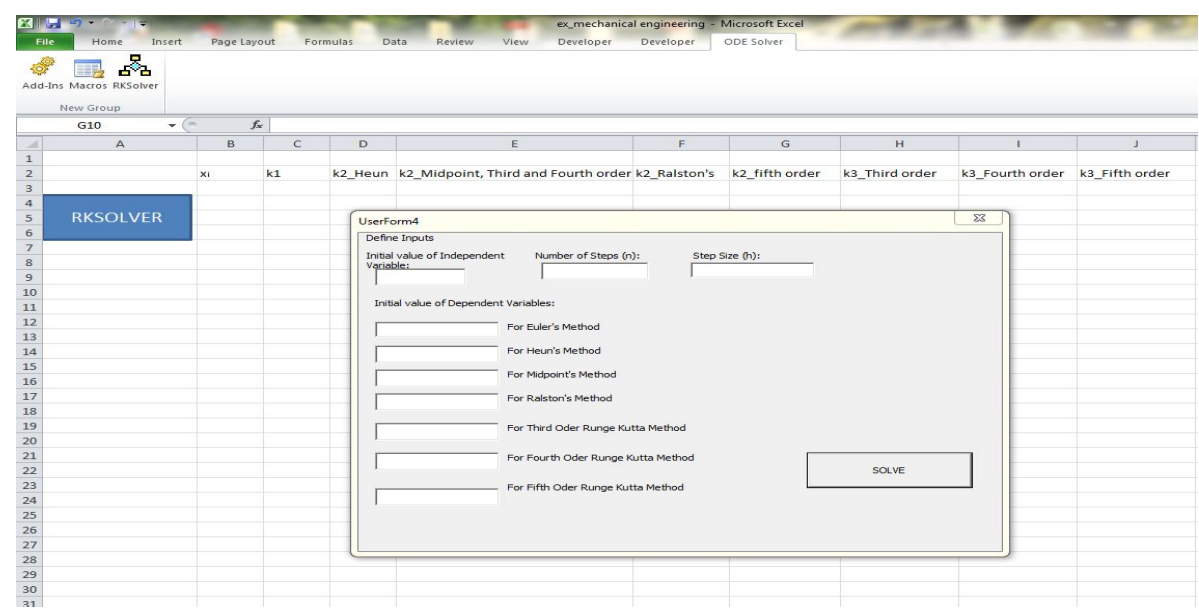

Figure 4.12: Userform in spreadsheet for mechanical engineering example

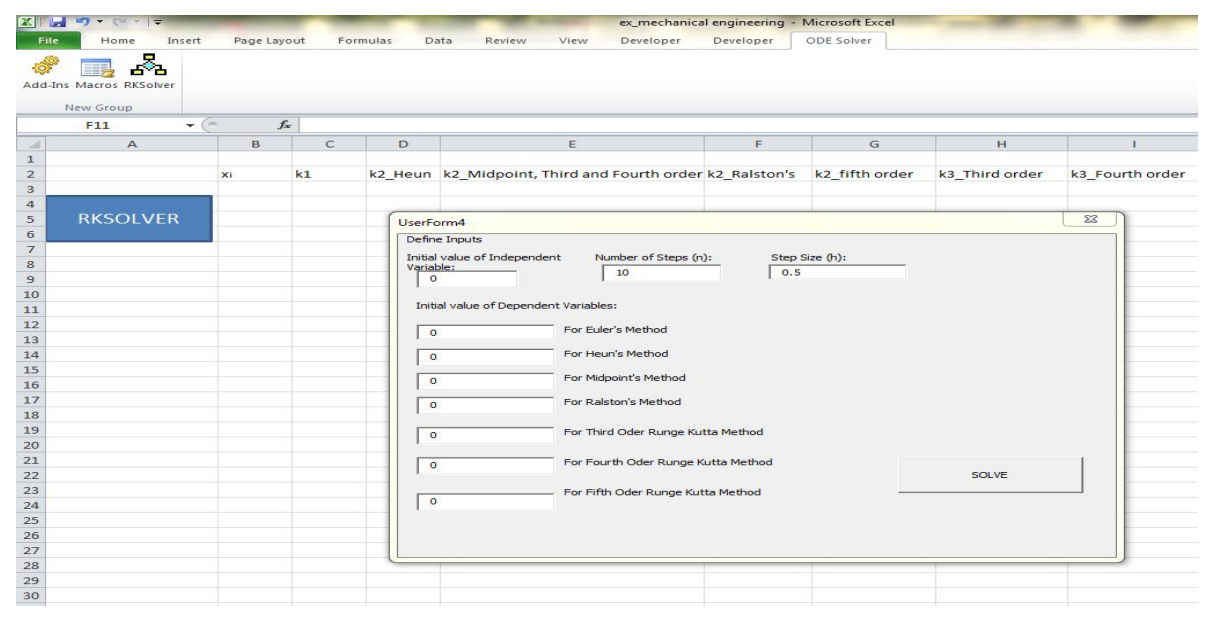

Figure 4.13: Filled userform for mechanical engineering example

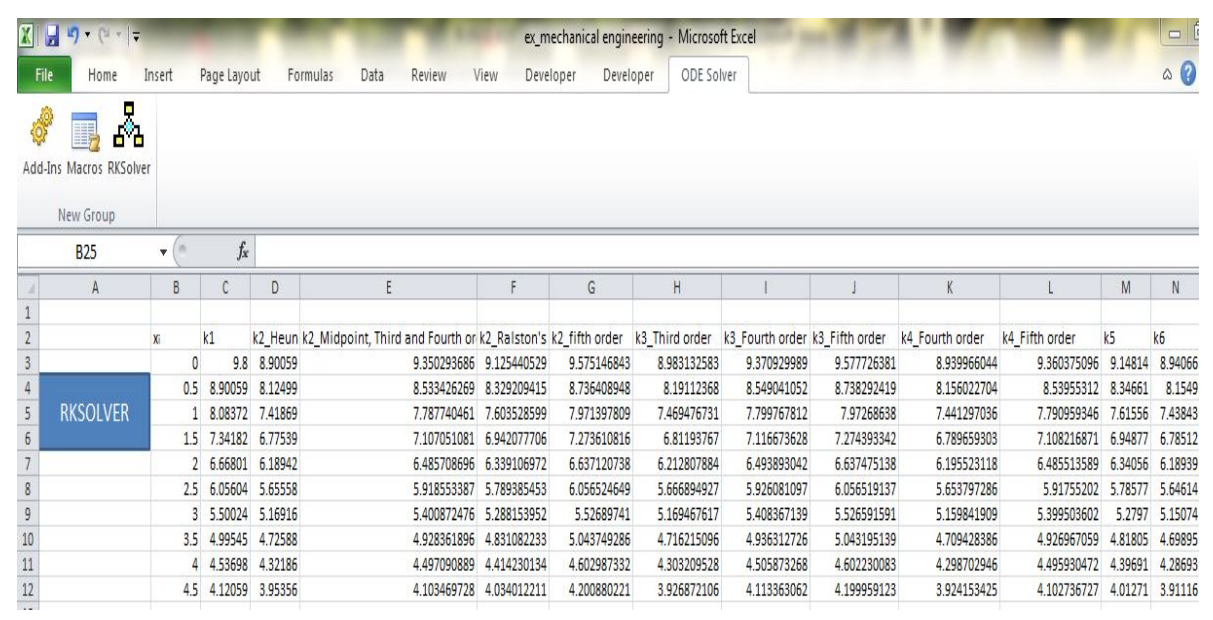

Figure 4.14: Computation results for k's for mechanical engineering example 


\begin{tabular}{|c|c|c|c|c|c|c|c|c|}
\hline & & & & ex_mec & Chanical engi & lineering - $M$ & icros & soft Excel \\
\hline Insert & Page $L$ & ayout & For & rmulas & Revi & Vien & & Developer \\
\hline $\mathrm{Cal}$ & libri (Body & - & 10 & $=\mathbf{A}^{-} \mathbf{A}^{-}$ & $\overline{\overline{\underline{\underline{ }}}} \equiv \overline{\overline{\underline{\underline{ }}}} \equiv$ & $\equiv \mid \geqslant-$ & 宣 & Wrap Text \\
\hline nter & $\underline{\mathbf{U}}$ & $-1 \pm$ & - & $\underline{\Delta}-\underline{A}$ & $\underline{\underline{\underline{\underline{\underline{\underline{1}}}}}} \overline{\overline{\underline{\underline{\underline{\underline{a}}}}}} \overline{\overline{\bar{z}}}$ & 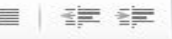 & 垔 & 긱 Merge \& Center \\
\hline 5 & & Font & & $\sqrt{x}$ & & Alignme & ent & \\
\hline$-c$ & & $f=$ & & & & & & \\
\hline 0 & $P$ & a & & $\mathbf{R}$ & s & $T$ & & $u$ \\
\hline yit1Euler & yit1Heun & yi+1Midpoi & sint & yi+1Ralston's & yitzThird Ord & yi+1Fourth Ord & & yit1Fifth Order \\
\hline 4.9 & 4.67515 & 4.67514 & 4684 & 4.67514684 & 4.6820256 & 4.681867 & 783 & 4.68187063 \\
\hline 9.35029 & 8.93154 & 8.94185 & 5998 & 8.93498121 & 8.9508103 & 8.950329 & 842 & 8.951759743 \\
\hline 13.3922 & 12.8071 & 12.8357 & 8302 & 12.8167774 & 12.842823 & 12.8419 & 993 & 12.84604052 \\
\hline 17.0631 & 16.3364 & 16.3892 & 2557 & 16.3544403 & 16.39132 & 16.39024 & 353 & 16.39786458 \\
\hline 20.3971 & 19.5508 & 19.6321 & 1101 & 19.5788115 & 19.626625 & 19.62547 & 184 & 19.63744819 \\
\hline 23.4251 & 22.4787 & 22.5913 & 3868 & 22.5179473 & 22.576388 & 22.57539 & 771 & 22.59233293 \\
\hline 26.1752 & 25.1461 & 25.291 & 1823 & 25.197372 & 25.265821 & 25.26527 & 779 & 25.28762321 \\
\hline 28.6729 & 27.5764 & 27.756 & 6004 & 27.6403069 & 27.717913 & 27.71812 & & 27.74620258 \\
\hline 30.9414 & 29.7911 & 30.0045 & 5494 & 29.8678802 & 29.953626 & 29.95493 & 061 & 29.98893081 \\
\hline 33.0017 & 31.8096 & 32.0562 & 2843 & 31.899316 & 31.992071 & 31.99479 & & 32.03482341 \\
\hline & & & & & & & & \\
\hline
\end{tabular}

Figure 4.15: Computation results for each RK method for mechanical engineering example

\begin{tabular}{|c|c|c|c|c|c|c|c|c|c|}
\hline \multicolumn{4}{|l|}{ rosoft Excel } & \multicolumn{3}{|c|}{ Chart Tools } & \multicolumn{3}{|c|}{ 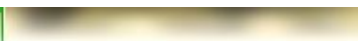 } \\
\hline \multicolumn{2}{|c|}{ Developer } & veloper & ODE Solver & \multicolumn{2}{|c|}{ Design } & Format & \multirow{3}{*}{\multicolumn{2}{|c|}{$\begin{array}{l}\text { Delete Format } \\
\text { Cells }\end{array}$}} & \multirow{3}{*}{$\begin{array}{l}\Sigma \text { Autost } \\
\text { (9) Fill - } \\
2 \text { Clear }\end{array}$} \\
\hline \multirow{2}{*}{\multicolumn{2}{|c|}{$\begin{array}{l}\text { Wrap Text } \\
\text { Merge \& Center - }\end{array}$}} & General & $=$ & & \multirow{2}{*}{$\begin{array}{c}\begin{array}{c}\text { Format } \\
\text { onditional } \\
\text { onmatting - as Table- } \\
\text { Styles }\end{array} \\
\end{array}$} & \multirow{2}{*}{ Cell } & & & \\
\hline & & $\begin{array}{l}\text { Numbe } \\
\text { Num }\end{array}$ & 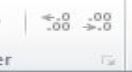 & & & & & & \\
\hline $\mathrm{v}$ & $\mathrm{w}$ & $x$ & r & & $z$ & $A A$ & $A B$ & & $A C$ \\
\hline TRUE Results & Error_Euler & Error_Heun & Error_midpoin & & Error_Ralstor & Error_third order & Error_fourth order & Error_t & fifth order \\
\hline 4.6818706 & 4.6590216 & $5 \quad 0.1436134$ & 0.14361 & 3389 & 0.1436134 & 0.003310099 & $6.09425 E-05$ & & $1.30578 \mathrm{E}-07$ \\
\hline 8.9531822 & 4.4354227 & 0.241706 & 0.126460 & 0405 & 0.2032908 & 0.026492481 & 0.031858677 & & 0.015887815 \\
\hline 12.849937 & 4.2196044 & $\begin{array}{ll}4 & 0.3330287\end{array}$ & 0.110560 & 0409 & 0.2580538 & 0.055359434 & 0.0617734332 & & 0.030324126 \\
\hline 16.404981 & 4.0114882 & 2. 0.4177613 & 0.09585 & 5366 & 0.3080802 & 0.083269244 & 0.089834125 & & 0.04337841 \\
\hline 19.648278 & 3.8109851 & 10.4960917 & 0.08228 & 7895 & 0.3535514 & 0.110203575 & 0.116073336 & & 0.055119649 \\
\hline 22.607167 & 3.617996 & 0.568215 & 0.0698 & 8014 & 0.3946518 & 0.136147269 & 0.140527115 & & 0.065616264 \\
\hline 25.306587 & 3.4324119 & \begin{tabular}{|l|l|} 
& 0.6343328
\end{tabular} & 0.058340 & 0185 & 0.4315674 & 0.161088317 & 0.163234757 & & 0.074935927 \\
\hline 27.769291 & 3.2541143 & $3 \quad 0.6946524$ & 0.047849 & 9573 & 0.4644862 & 0.185017819 & 0.184238583 & & 0.083145376 \\
\hline 30.016038 & 3.0829762 & 0.7493857 & 0.038276 & 6016 & 0.49359666 & 0.207929928 & 0.203583685 & & 0.090310244 \\
\hline 32.065765 & 2.918862 & 0.7987488 & 0.029567 & 7196 & 0.5190872 & 0.229821773 & 0.221317681 & & 0.0964948889 \\
\hline & & & & & & & & & \\
\hline & & & & & & & & & \\
\hline
\end{tabular}

Figure 4.16: Computation results for exact results (true results) and true percent relative errors of each RK method for mechanical engineering example

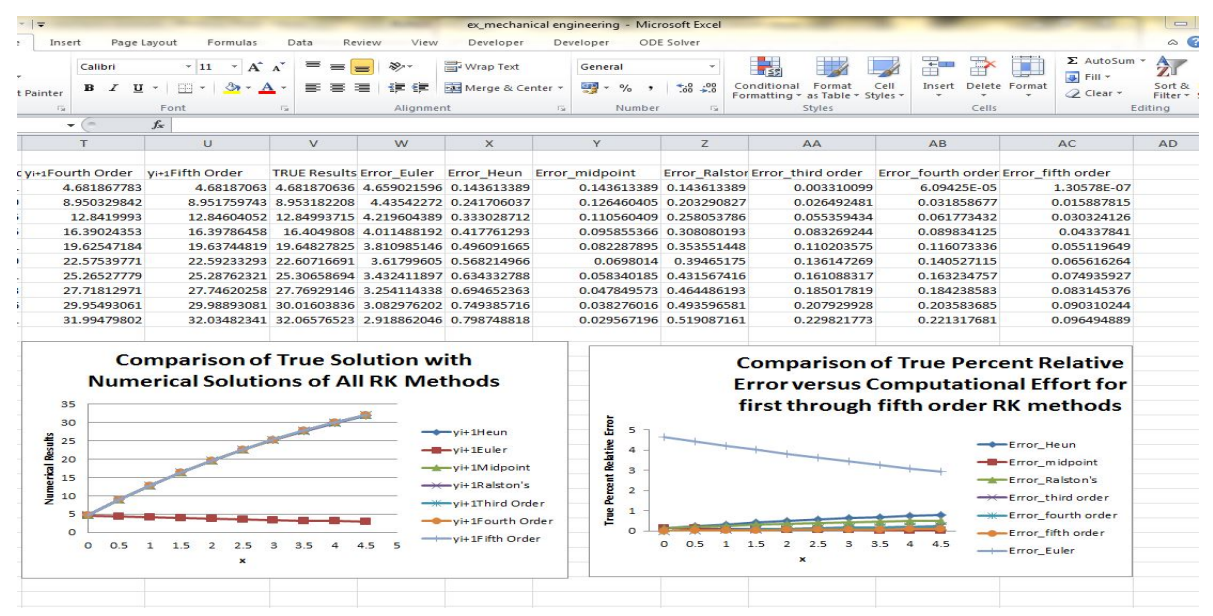

Figure 4.17: Graphical display of the computation results for mechanical engineering example 


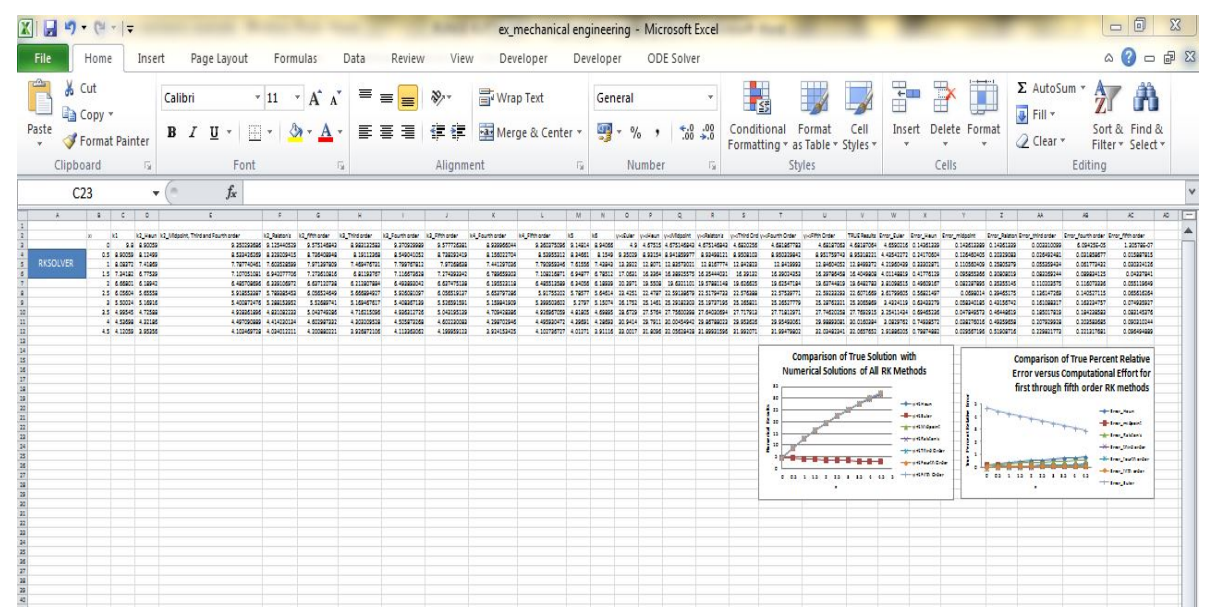

Figure 4.18: The spreadsheet image of full computation results for mechanical engineering example

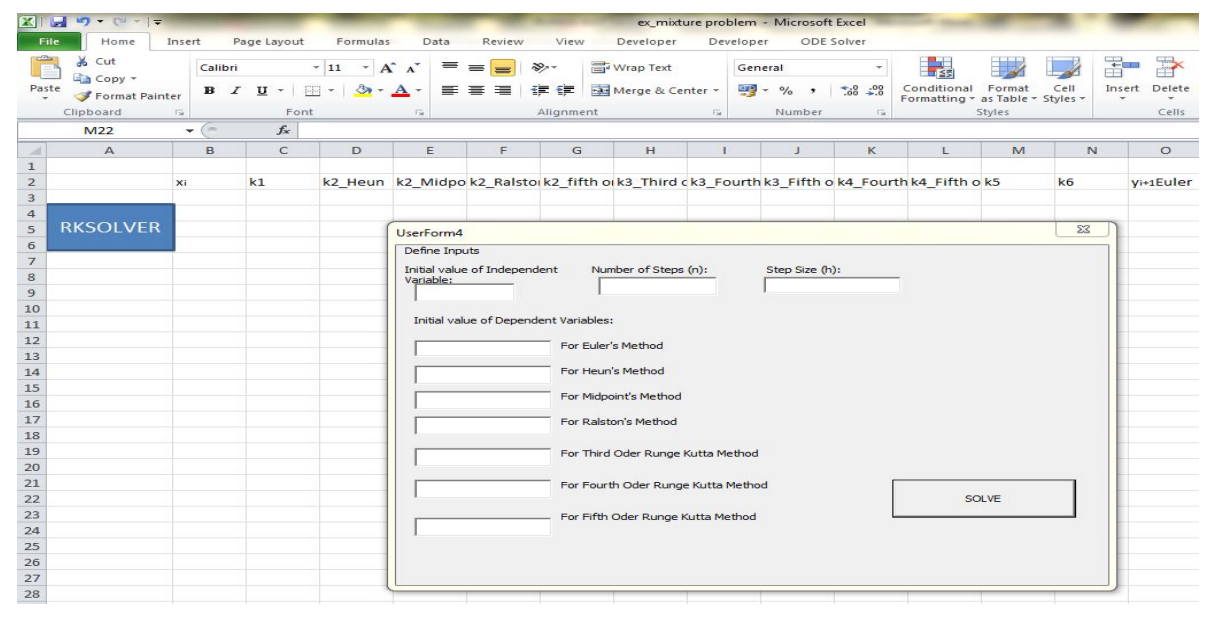

Figure 4.19: Userform in spreadsheet for mixture problem

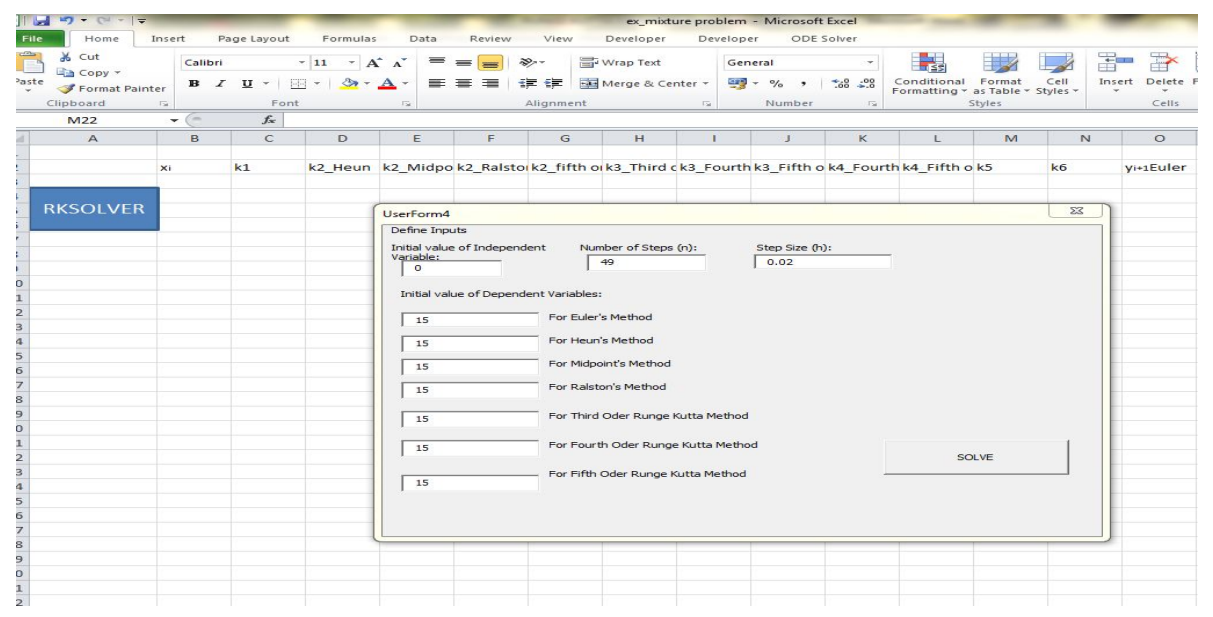

Figure 4.20: Filled userform for mixture problem 


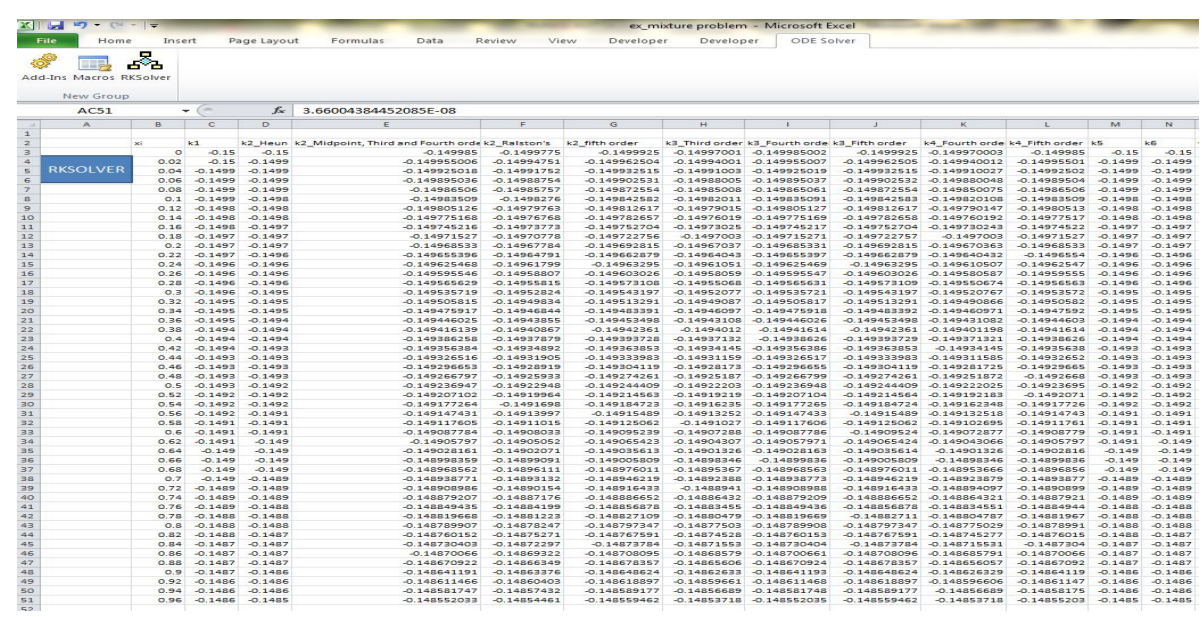

Figure 4.21: Computation results for k's for mixture problem

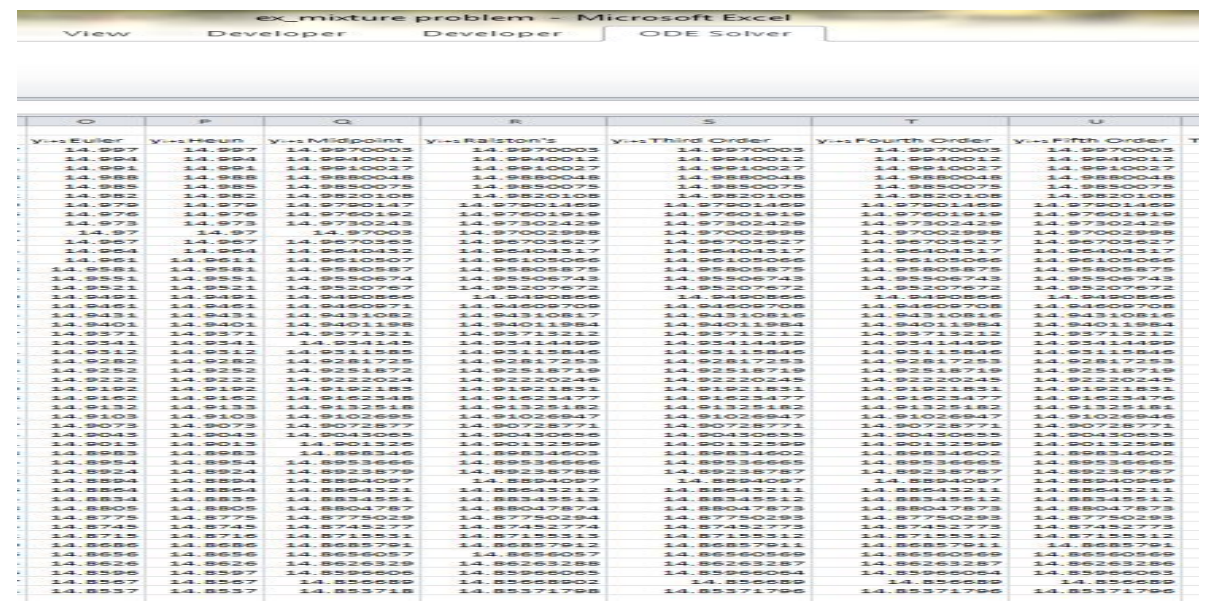

Figure 4.22: Computation results for each RK method for mixture problem

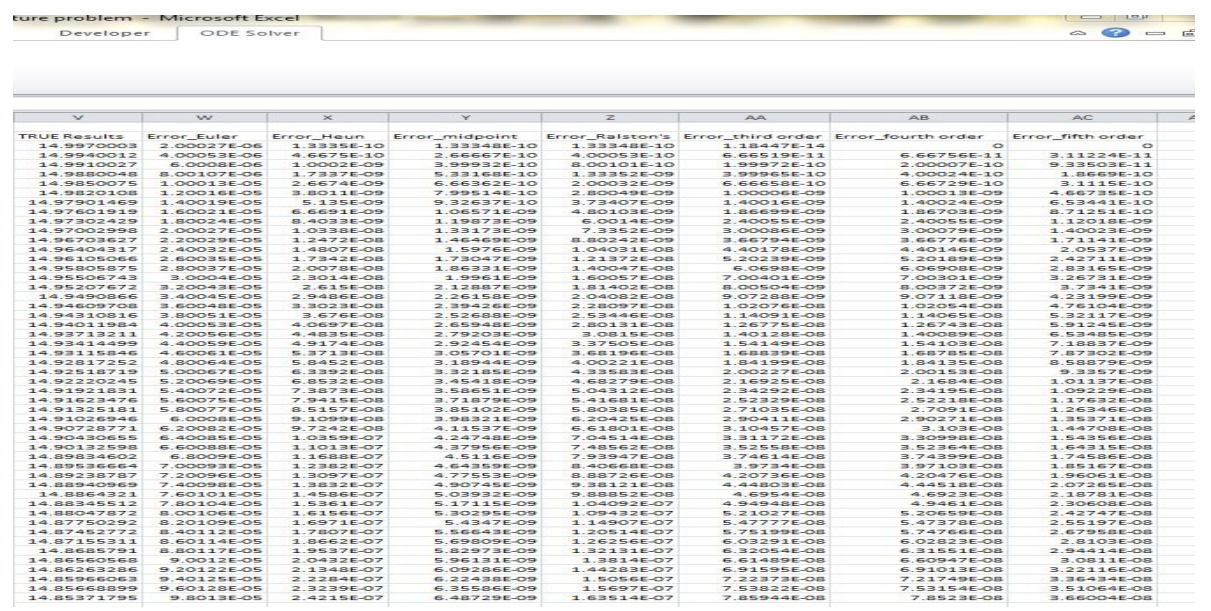

Figure 4.23: Computation results for exact results (true results) and true percent relative errors of each RK method for mixture problem 


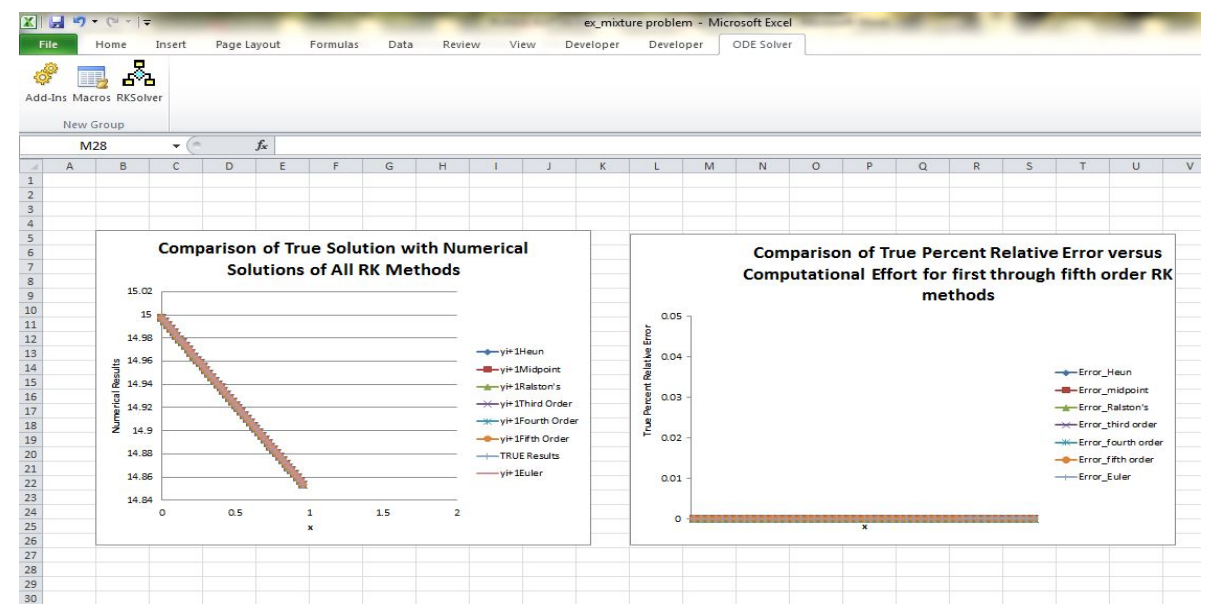

Figure 4.24: Graphical display of the computation results for mixture problem

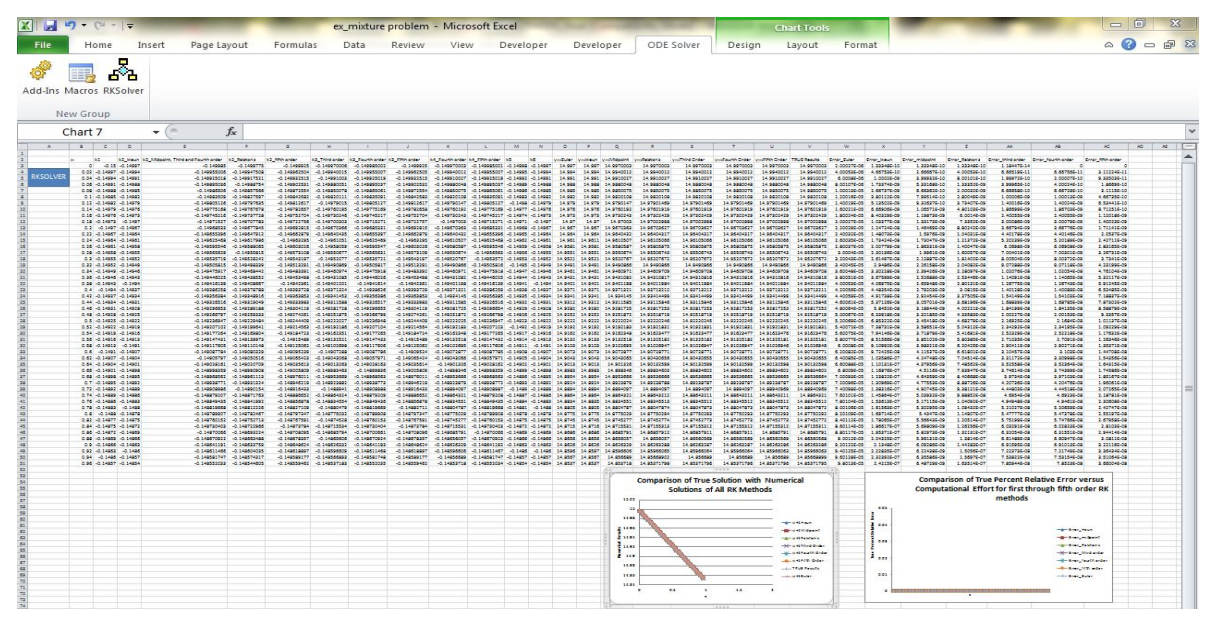

Figure 4.25: The spreadsheet image of full computation results for mixture problem 
This spreadsheet solver is so user-friendly that users (students, educators and also beginner users of Excel and VBA) only require to click RKSOLVER button and enter relevant information in userform to perform all computations for the complete solution of IVPs efficiently without typing any commands in the spreadsheet.

It is hoped that this spreadsheet solver can be used as a marking scheme for users who need the complete solutions of IVPs numerically and analytically with comparison of them in terms of error at the same time. Lastly, it is hoped that this spreadsheet solver could serve as not only a numerical IVP tool but also an analytical IVP tool with a comparison of them that is convenient for the community of engineering educators and students.

\section{References}

[1] S.C. Chapra, R.P. Canale, Numerical Methods for Engineers, McGraw Hill, 2006

[2] D.G. Lilley, Numerical Methods Using Excel/VBA for Engineers, Cambridge University Press, 2010.

[3] E.D. Laughbaum, K. Seidel, Business Math Excel Applications, Prentice Hall, 2008.

[4] R.W. Larsen, Engineering with Excel, Pearson Prentice Hall, 2009.

[5] D.M. Bourq, Excel Scientific and Engineering, Cookbook. O'Reilly, 2006.

[6] E. J. Billo, Excel for Scientists and Engineers, Wiley -Interscience, 2007.

[7] S.C. Chapra, Power Programming with VBA/Excel, Prentice Hall, Upper Saddle River, 2003.

[8] S.L. Kek, K.G. Tay, Design of spreadsheet solver for polynomial interpolation, National Seminar on Science and Technology (PKPST 2009), 69-73 (2009).

[9] K.G. Tay, S.L. Kek, R. Abdul-Kahar, A bivariate approximation spreadsheet calculator by Lagrange interpolation, Spreadsheets in Education (eJSiE), 7 , 1-8 (2014).

[10] S.L. Kek, K.G. Tay, Solver for system of linear equations, Proceeding of the National Symposium on Application of Science Mathematics 2008 (SKASM 2008), 605-615 (2008).

[11] K.G. Tay, S.L. Kek, R. Abdul-Kahar, Solving non-linear systems by Newton's method using spreadsheet Excel, Proceeding of the 3rd International Conference on Science and Mathematics Education (CoSMED 2009), 452-456 (2009).

[12] K.G. Tay, S.L. Kek, Approximating the dominant eigenvalue using Power method through spreedsheet Excel, Proceeding of the National Symposium on Application of Science Mathematics 2008 (SKASM 2008), 599-604 (2008).

[13] K.G. Tay, S.L. Kek, Approximating the Smallest Eigenvalue Using Inverse Method Through Spreadsheet Excel, Proceeding of the 17th National Symposium on Mathematical Science (SKSM 2009), 653-658 (2009).

[14] K.G. Tay, S.L. Kek, R. Abdul-Kahar, Improved Richardson's Eextrapolation spreadsheet calculator for numerical differentiation, AIP Conference Proceedings, 1605, 740-743 (2014).

[15] K.G. Tay, S.L. Kek, R. Abdul-Kahar, M.A. Azlan, M.F. Lee, A Richardson's extrapolation spreadsheet calculator for numerical differentiation, Spreadsheets in Education (eJSiE), 6, 1-5 (2013).

[16] K.G. Tay, S.L. Kek, R. Abdul-Kahar, Numerical differentiation spreadsheet calculator, Proceedings of the National Symposium on Application of Science Mathematics 2008 (SKASM 2013), 111-120 (2013).

[17] C.K. Ghaddar, Unlocking the spreadsheet utility for calculus: A pure worksheet solver for differential equations, Spreadsheets in Education (eJSiE), 9, $1-16(2016)$.

[18] K.G. Tay, S.L. Kek, R. Abdul-Kahar, A spreadsheet solution of a system of initial value problems using the fourth-order Runge-Kutta method, Spreadsheets in Education (eJSiE), 5, 1-10, (2012).

[19] K.G. Tay, S.L. Kek, Fourth Order Runge-Kutta Method Using Spreadsheet Excel. Proceedings of the 4th International Conference on Research and Education in Mathematics (ICREM4). 666-672 (2009).

[20] K.G. Tay, T.H. Cheong, M.F. Lee, S.L. Kek, R. Abdul-Kahar, A fourth-order Runge-Kutta (RK4) spreadsheet calculator for solving a system of two first-order initial value problems using Visual Basic (VBA) Programming, Spreadsheets in Education (eJSiE), 8, 1-9 (2015).

[21] K.G. Tay, S.L. Kek, T.H. Cheong, R. Abdul-Kahar, M. F. Lee, The fourth order Runge-Kutta spreadsheet calculator using VBA programming for initial value problems, Procedia-Social and Behavioral Sciences, 204, 231-239 (2015). 